\title{
Special issue of the Knowledge Representation and Reasoning Track, Symposium on Applied Computing (SAC) 2017
}

\author{
Preface
}

This special issue of Fundamenta Informaticae publishes extended and revised versions of the best papers presented at the 1st edition of the track on Knowledge Representation and Reasoning (KRR), part of 32nd Annual ACM Symposium on Applied Computing (SAC). ${ }^{1}$ This event represents the first edition of such a track; its success has led to the 2018 and and the 2019 editions

The topics of the track cover an important field of research in Artificial Intelligence. Knowledge Representation and Reasoning (KRR) is dedicated to representing information about the world in a form that a computer system can utilise to solve complex tasks. Examples of knowledge representation formalisms include semantic nets, systems architecture, frames, rules, and ontologies. Some examples of automated reasoning engines include inference engines, theorem provers, and classifiers. KRR track is a venue for all the researchers and practitioners working on the fundaments and applications of reasoning, and cross-fertilisation among different areas (e.g., Argumentation and Belief Revision).

Knowledge-representation and Reasoning (KRR) is the field of artificial intelligence that focuses on designing computer representations that capture information about the world that can be used to solve complex problems. Its goal is to understand and build intelligent behaviour from the top down, focusing on what an agent needs to know with the purpose to behave intelligently, how this knowledge can be represented symbolically, and how automated reasoning procedures can make this knowledge available as needed. In KRR, a fundamental assumption is that an agent's knowledge is explicitly represented in a declarative form, suitable for processing by dedicated reasoning engines. Topics of interest include, but are not limited to: argumentation, belief revision and update, belief merging, commonsense reasoning, contextual reasoning, description logics, diagnosis, abduction, explanation, inconsistency and exception tolerant reasoning, para-consistent logics, KR and autonomous agents (intelligent agents, cognitive robotics, multi-agent systems), KR and decision making - game theory - social choice, KR and machine learning - inductive logic programming - knowledge discovery and acquisition, logic programming, answer set programming, constraint (logic) programming, non-monotonic logics, default logics, conditional logics, preferences (modelling and representation, preference-based reasoning), reasoning about knowledge and belief, dynamic epistemic logic, epistemic and doxastic logics, reasoning systems and solvers, knowledge compilation, spatial reasoning

\footnotetext{
${ }^{1}$ http://www.dmi.unipg.it/bista/organizing/KR@sac2017/.
} 
and temporal reasoning, qualitative reasoning, uncertainty, representations of vagueness (many-valued and fuzzy logics). The aim of the conference track is fostering the cross-fertilisation of ideas stemming from all these different but related areas.

Concerning the 2017 edition (first edition), 9 papers were presented at KRR (990 in all the 38 tracks of SAC). After the conference, authors of selected papers had the possibility to submit extended versions of their works for possible publication in this special issue. After two further rounds of reviews, the following two papers were finally accepted:

- Candidate Selection and Instance Ordering for Realtime Solver Configuration by Tadhg Fitzgerald and Barry O’Sullivan.

- A Reconstruction of Default Conditionals within Epistemic Logic by Costas D. Koutras, Christos Moyzes, and Christos Rantsoudis.

Acknowledgments. We would like to thank the authors who submitted their articles to this special issue. The submissions were impressive both in quantity and quality.

Our sincere appreciation goes to the anonymous reviewers that evaluated the papers submitted to this issue. We also would like to express our gratitude to Damian Niwinski, Editor-in-Chief of Fundamenta Informaticae, for this possibility and for his support throughout the whole process.

Finally, we are very grateful to the Program Committee members and to the external anonymous reviewers of KRR 2017 for their work, as well as to the authors and the participants that took part in the conference track.

Editors of this special issue

Stefano Bistarelli

DMI, Università di Perugia, Italy

stefano.bistarelli@dmi.unipg.it

Francesco Santini

DMI, Università di Perugia, Italy

francesco.santini@unipg.it

Paolo Torroni

DISI, Università di Bologna, Italy

paolo.torroni@unibo.it 